\title{
Bio-Machining: Efficient Machining of Smart Micro-device in Health Monitoring and Prognosis
}

\author{
Ashwani Singh and Navin Mishra* \\ ${ }^{1}$ Department of Mechanical and Industrial Engineering, IIT Delhi, New Delhi, India \\ ${ }^{2}$ Department of Mechanical and Industrial Engineering, IIT Roorkee, Roorkee, Uttarakhand, India
}

\begin{abstract}
With the advent of Nano Technology Health Care is being redefined with the help of smart micro-devices both for health monitoring and administering drugs. This article looks into the efficiency of the current micro and nano devices and explores the possibility of bio-machining as an alternate to current micro-machining technologies as an efficient and scalable alternative. There is limited research in the field of bio-machining and its application is limited.
\end{abstract}

Keywords: Health care; Smart micro-devices; Micromachining; Bio machining

\section{Introduction}

\section{Healthcare costs}

Healthcare costs are increasing and the world population is ageing, there is a need to monitor a patient's health status while he is out of the hospital in his personal environment [1]. The proportion of young workers in developed countries has been shrinking. Elderly people have a higher level of disability due to age-related diseases, a greater need for care and assistance and are more likely to be admitted to a hospital or nursing home. Permanent admission to a care home is an expensive way of providing care for elderly, most of whom prefer to remain in their own home [2,3]. Data on health from 30 countries of the organization for Economic cooperation and Development show that health care expenditures as a proportion of GDP are at an all-time high, due to both increased expenditures and to a general economic slowdown. France spent $11 \%$ of its GDP on healthcare delivery in 2015 according to OECD statistics, the USA about $16.9 \%$ which is also the highest in the world, Canada and Switzerland are spending around $11.4 \%$ of their GDP respectively $[4,5]$.

\section{Telecare, Telehealth and Telemedicine}

Telecare, Telehealth and Telemedicine are new models of care already in use, bringing solutions to healthcare issues. The role of Smart wearable system is to match the living environment with the physical and cognitive abilities and limitations of those suffering from disabilities or disease, thereby enhancing performance and minimising the risk of illness, injury, and inconvenience. These systems support independent living for the elderly, post-operative rehabilitation for patients to expedite recovery and assessment or enhancement of individual sportive or technical abilities [6]. Smart Wearable System may include a wide range of wearable or implantable devices, including sensors, actuators, smart fabrics, power supplies, wireless communication networks, processing units, multimedia devices, user interfaces, software and algorithm for data capture ,processing and decision support. These systems are able to measure vital signs, such as body and skin temperature, heart rate, arterial blood pressure, blood oxygen saturation, electrocardiograms (ECG), electroencephalograms (EEG), and respiration rate $[7,8]$. The measurements are forwarded via a wireless sensor network either to a central connection node, such as a personal digital assistant or directly to a medical centre. The physician can then manage the patient based on the transmitted data. The patient wears the SWS such that the medical professionals monitor the patient in real-time for longer periods than are possible during a hospital stay or a visit, the importance of SWS seems higher in developing countries where the healthcare facilities are limited and doctors to patient ratio is poor. SWS system can even issue alerts in the event of an emergency. During a change in climate one is prone to infections for example during summers one is prone to have a lung infection, dehydration, however, it can be avoided if the patient's behaviour as a result of disease are collected early and adequate preventive cure is suggested or initiated before the situation becomes dangerous [9].

\section{Bio-therapeutic drugs}

Bio-therapeutic drugs such as peptides, proteins, DNA and RNA are large molecules that cannot be easily administered orally or through transdermal means. Nanotechnology is providing new tools and materials to probe and manipulate biological processes at the Nanoscale (1-100 nm) [10,11]. One-dimensional Nanomaterials are being used as intracellular biosensors, drug delivery carriers and imaging agents. Further, there has been a rapid development of microfluidics-based industry including the expansion of the use of micro-dispenser, Drug Delivery Systems (DSSs) and biochips warranting increased demand for micro and Nanoneedles. For the treatment of dermatological conditions such as psoriasis and cosmetic applications, micro rollers have been developed for drug delivery into large areas of skin simultaneously and without pain. Typically these micropumps are micro-electrochemical systems (MEMS) devices which are integrated with other systems on a single chip for controlled drug delivery system [12]. Advances in the field of MEMS have addressed a number of clinical indications such as drug release and biosensors for point of care testing. MEMS devices including micro-reservoir drug depots, micropumps, valves, and sensors, have been developed for the delivery of microgram quantities of drugs, biosensors and other features that are responsive to the local device environment can enable MEMS to function in an integrated manner with its biological surroundings $[13,14]$.

*Corresponding author: Navin Mishra, Department of Mechanical and Industria Engineering, IIT Roorkee, Roorkee, Uttarakhand, India, Tel: 9829104187; E-mail: chtainia@yahoor

Received January 16, 2017; Accepted March 04, 2017; Published March 14 2017

Citation: Singh A, Mishra N (2017) Bio-Machining: Efficient Machining of Smart Micro-device in Health Monitoring and Prognosis. J Biosens Bioelectron 8: 240. doi: 10.4172/2155-6210.1000240

Copyright: ( 2017 Singh A, et al. This is an open-access article distributed under the terms of the Creative Commons Attribution License, which permits unrestricted use, distribution, and reproduction in any medium, provided the original author and source are credited. 
Micro and nanoneedles: Microneedles have been used mainly for drug delivery into the skin [15]. Recent research has opened the window for microneedles to be used as diagnostic tool for extraction of analytes from the skin for ex-vivo analysis. Further advancement has been made into integrating sensors onto the microneedles for in situ sensing in the skin, lactates, dissolved oxygen and glutamate [16]. These microneedles have also been used as bio-electrical interfaces for neural recording and stimulation, measurements in ECG and EEG. Beyond intracellular delivery, Nano needles are being used in biological experiments in living cells. These are made electrically conductive and being fabricated as a probe to measure electrochemical reactions and signal processing process inside cells. Presently glass, silicon and polymers are used as primary material for microneedles. Micro and nano needles fabricated with glass and silicon have high mechanical strength but are brittle in nature making them vulnerable to bending and shock effect. Polymers offer low production cost and ease of fabrication but have relatively low mechanical strength. Considering metals for micro and nano needles, they exhibit good mechanical properties (toughness, strength, thermal conductivity) and electrical conductivity. As per research data available, metal microneedles have been prepared by three-dimensional ablation, laser cutting, wet etching and metal electroplating methods. Metal microneedles have been fabricated as solid and hollow types. The solid metal microneedles tend to be very strong whereas hollow metal microneedles are susceptible to fracture.

Metal micro pumps: In microfluidic systems, the target applications include chemical and biological analysis, sensing and drug delivery. A microfluidic system is one in which fluid flows in miniature devices at micro and nano level. Micro level tubes have been fabricated by conventional processes such as extrusion, drawing and the nontraditional process of EDM [17]. Mechanical micro pumps use the motion of a solid such as gear or diaphragm or a fluid to generate the pressure difference to move the fluid. At present, these pumps are being fabricated of polymers and metals using conventional processes. Shape Memory Alloy (SMA) micro pumps are metals that show two unique properties such as pseudoelasticity and shape memory. The diaphragm of SMA micropumps is mostly made of titanium and nickel alloy due to their high force-to-volume ratio and high damping capacity [18].

Micro-needle-rollers: Cosmetic applications and dermatological conditions such as psoriasis require drug delivery into large areas. To address this issue, microneedle rollers were introduced. These are mounted on a surface generally cylindrical and rolls on the skin such that each micro-needle may pierce the skin multiple times. As per research data available, microneedle rollers have been fabricated with stainless steel which has received the most attention. The disadvantage is that they are not cost effective and as multiple use devices require regular cleaning [19].

\section{Miniaturisation}

Miniaturisation is the key to success of any product being manufactured but it presents its own set of challenges to scientists and engineers. Microfabrication process is applied in various industrial micro-applications such as electronics, optics, aerospace and biotechnology. Reliability and repeatability are two major aspects of these processes. The present processes of micromachining include micro-milling, micro-EDM (Electro Discharge Machining) and chemical etching [20]. The biggest problem associated with these techniques is the HAZ on the material being machined affecting the physical and chemical properties. The reliability is often undermined especially in the case of implantable devices. Bio-machining can be considered as a viable option for fabrication of micro and Nano needles based on metals since this technology is environment-friendly and has the potential for scaling up as required. The disadvantage as of now is that Bio-machining has been only studied for iron and copper whereas microneedles have been fabricated with stainless steel and titanium metal sheets and nickel. Bio-machining can suitably be used to fabricate the gears of the micropumps which have been demonstrated wherein a gear shape was obtained from copper with tooth depth of $45 \mu \mathrm{m}$.

Bio-machining: It is a process in which micro-organisms attack the material in a controlled environment which produces an environmentfriendly, energy efficient and cost effective approach for material removal. There are three basic principles of Bio-machining (i) Redox reaction (ii) Formation of organic and inorganic acids (iii) Excretion of complexing agents. CAD software may be used to design the final product on the workpiece [21]. After the formation of the photoresist masked layer, the material is kept in a bacteria cultured solution (with or without the presence of bacteria) for the removal of bacteria as per the principles defined earlier. The experiments conducted by researchers have been based on Acidic Thiobacillus Ferrooxidans/Thiooxidans.

\section{Advantages of Bio-machining}

The major advantage of using Bio-machining as micromachining process is no Heat affected Zone (HAZ), environmental friendly and can be scaled up for mass production [22]. Further, there is no tool wear as it can be recycled/regenerated continuously. The bacteria used as a tool has a size of $1 \mu \mathrm{m}$, thus theoretically machining resolution of this size is possible, however, in experiments, the $3 \mu \mathrm{m}$ resolution has been achieved. The disadvantages associated with this process is that the bacteria used for machining grows only in acidic $\mathrm{pH}$, slow process, culturing of bacteria under controlled conditions is time-consuming and research has undertaken only on materials such as iron and copper.

\section{Conclusion}

Fabrication of a metal based micro-feature through bio-machining is still in nascent stage and research is underway to optimise the process and make it suitable for mass production. Due to global concern on environmental effects of machining and adoption of green technologies, there exists a bright future for non-traditional processes such as biomachining which are eco-efficient and reduce the unwanted outputs and can be recycled.

\section{References}

1. Mehrotra $P$ (2016) Biosensors and their applications: A review. Journal of Oral Biology and Craniofacial Research 6: 153-159.

2. Hammond J, Formisano N, Estrela P, Carrara S, Tkac J (2016) Electrochemica biosensors and nano biosensors. Essays in Biochemistry 60: 69-80.

3. Gonzalez-rodriguez J, Raveendran M (2015) Importance of Biosensors. Biosensors J 4: e104.

4. Mehrvar M, Abdi M (2004) Recent Developments, Characteristics, and Potential Applications of Electrochemical Biosensors. Analytical Sciences 20 1113-1126.

5. Chan M, Estève D, Fourniols JY, Escriba C, Campo E (2012) Smart wearable systems: Current status and future challenges. Artificial Intelligence in Medicine 56: $137-156$

6. Pantelopoulos A, Bourbakis NG (2010) A Survey on Wearable Sensor-Based Systems for Health Monitoring and Prognosis. IEEE Transactions on Systems, Man, and Cybernetics 40: 1-12.

7. Suwandi D, Whulanza Y, Istiyanto J (2014) Visible Light Maskless Photolithography for Bio machining Application. Applied Mechanics and Materials 493: 552- 557

8. Ikeda S, Kakinuma Y (2011) Basic Study of Nanofabrication of Biodegradable 
Citation: Singh A, Mishra N (2017) Bio-Machining: Efficient Machining of Smart Micro-device in Health Monitoring and Prognosis. J Biosens Bioelectron 8: 240. doi: 10.4172/2155-6210.1000240

Page 3 of 3

Plastics applying Biochemical Machining. Precision Engineering 35: 440-446.

9. Teo MA, Shearwood C, Ng KC, Lu J, Moochhala S (2005) In Vitro and In Vivo Characterisation of MEMS Microneedles. Biomedical Microdevices 7: 47-52.

10. Istiyanto J, Saragih AS, Ko TJ (2012) Metal based Micro- feature Fabrication using Bio machining Process. Microelectronic Engineering 98: 561-565.

11. Park JH, Choi SO, Seo S, Choy YB, Prausnitz MR (2010) A Microneedle Roller for Transdermal Drug Delivery. European Journal of Pharmaceutics and Biopharmaceutics 76: 282-289.

12. Kim YC, Park JH, Prausnitz MR (2012) Microneedles for Drug and Vaccine Delivery. Advanced Drug Delivery Reviews 64: 1547-1568.

13. Abrahi F, Haslina Jz, Yunus NM (2012) A Comprehensive Study of Micropumps Technology. Int J Electrochem Sci 7: 9765-9780.

14. Jung PG, Lee TW, Oh DJ, Ko SJ (2008) Nickel Microneedles Fabricated by Sequential Copper and Nickel Electroless Plating and Copper Chemical Wet Etching. Sensors and Materials 20: 045-053.

15. Istiyanto J, Kim MY, Ko TJ (2011) Profile Characteristics of Biomachined Copper. Microelectronic Engineering 88: 2614-2617.
16. Whulanza Y, Nadhif HM, Istiyanto J, Bachtiar BM (2016) PDMS Surface Modification using Bio machining Method for Biomedical Application. Journal of Biomimetics, Biomaterials and Biomedical Engineering 26: 66-72.

17. Xiaomeng li, Xiaoqing Wu, Peng Shi, Zuo-Guang Ye, et al. (2016) Lead-Free Piezoelectric Diaphragm Biosensors based on Micromachining Technology and Chemical Solution Deposition. Sensors 16: 69.

18. Zhu MW, Li HW, Chen XL, Tang YF, Lu MH, et al. (2009) Silica Needle Template Fabrication of Metal Hollow Microneedle Arrays. J Micromech Microeng.

19. Mishra S, Yadav M (2009) Bio machining - A Review. ELK Asia pacific Journals.

20. Kaneeda T, Yokomizo S, Miwa A, Morioka H (1997) Biochemical MachiningBiochemical Removal Process of Plastic. Precision Engineering 21: 57-6.

21. Torsi L (2013) Special Issue on Organic Electronic Bio-Devices. Biosensors 3: $116-119$.

22. Shikata S, Sreekumari KR, Nandakumar K, Ozawa M, Kikuchi Y (2009) Laboratory studies on Bio machining of Copper using Staphylococcus $\mathrm{sp}$. Biofouling, 25: 557-562. 